\title{
Testing a preference inference account of classic preference reversals
}

\author{
Harish Balakrishnan (harishb@iitk.ac.in) \\ Interdepartmental Programme in Cognitive Science, IIT Kanpur \\ Nisheeth Srivastava (nsrivast @cse.iitk.ac.in) \\ Department of Computer Science, IIT Kanpur
}

\begin{abstract}
Different theories of decision making assume different sources of preferences for the choices we make. A recent theory has proposed that preferences are inferred from past choices in respective contexts, and that such preference inference offers a rational basis for the origin of classic preference reversals given certain sequences of observations. In this paper, we empirically test this theory for some of the classic preference reversals - the attraction effect and the compromise effect, through longitudinal preferential choice experiments. In this paradigm, participants provide their preferences for different choices occurring in different contexts throughout the experiment. Results indicate evidence in favour of a considerable attraction effect but are mixed for a compromise effect. We briefly discuss the theoretical implications of our findings and possible directions for future work.

Keywords: decision making; inference; preference reversals; attraction effect; compromise effect; longitudinal preferential choice experiment paradigm
\end{abstract}

\section{Introduction}

Decision making is a complex cognitive process that is inherently context dependent. As best described by Slovic (Slovic, 1995):

“...decision making is a highly contingent form of information processing that is sensitive to task complexity, framing of the task, time pressure, response mode, reference points, and numerous other contextual factors..."

Different theories explain decision making from different perspectives and make different assumptions to explain the decision process. Descriptive theories such as the prospect theory (Kahneman \& Tversky, 1979) explains how people make decisions under uncertain circumstances or under risk. Normative theories of decision making prescribe how people should make decisions (Hickson \& Khemka, 2014) and posit that people choose options that maximizes their preferences. These preferences are often encoded as utility values, which are absolute numerical representations for preferences. Recently, it has been shown that living organisms, such as slime mold (Physarum polycephalum), use comparative valuation strategies rather than absolute valuation strategies, which require numerical value representations of preferences (Latty $\&$ Beekman, 2011). In any case, it is assumed that decision making is presupposed by the existence of preferences. But the question of where do these preferences come from? has generally been avoided. Different models of decision making have their own set of assumptions that suggest different sources for preferences. The associative-value based model (Bhatia, 2013), a model influenced by MDFT(multialternative decision field theory), uses a feed-forward neural network, assumes associative connections between attributes and alternatives in a context and suggests that preferences are accumulated over the course of the decision time. The Bayesian model of context sensitive value (Rigoli, Mathys, Friston, \& Dolan, 2017) assumes preferences are numerical values that arise from a precision-weighted prediction error and this naturally mandates feedback for the decisions made. Prior work (Srivastava \& Schrater, 2012, 2015), has suggested that preferences are inferred from prior history of choices made in corresponding contexts and a model of decision making is proposed based on Bayesian learning that doesn't necessitate preferences being mapped onto numerical values. An immediate prediction of this theory is that preference reversals can be elicited by varying the frequency of comparison presentations of choices in appropriate contexts.

The aim of this paper is to obtain empirical evidence for the above prediction by testing for some of the classic preference reversals - the attraction effect and the compromise effect. The following sections describe the experiments done, their results and the scope for future work.

\section{Methods}

Different placements of decoy with respect to the target and competitor options result in different kinds of preference reversals (figure 5). In all the experiments, A was the target, $\mathrm{B}$ was the competitor and $\mathrm{C}$ was the decoy. A longitudinal preferential choice experiment paradigm was used (figure 1).

\section{Attraction Effect}

Phase 1 was divided into two blocks in experiment $1 \mathrm{a}$ and $1 \mathrm{~b}$. Participants were encouraged to take a short break between the blocks. They were asked for their baseline preferences after every five animations or trials.

Experiment 1a The attraction effect was first tested with stimuli having a single attribute. Each participant witnessed simulations of football matches between four teams - A, B, C and $\mathrm{D}$. Block 1 consisted of sets of ten matches of A versus B and $C$ versus D. Similarly, block 2 had sets of ten matches of $A$ versus $C$ and $B$ versus $D$. The order of the sets within each block and the matches within each set were randomized. In phase 2, participants were asked for their final preferences in the form of the four choice sets containing two options each - $\{\mathrm{A}$ or $\mathrm{B}\},\{\mathrm{C}$ or $\mathrm{D}\},\{\mathrm{A}$ or $\mathrm{C}\},\{\mathrm{B}$ or $\mathrm{D}\}$. The order of presentation of these four choice sets were also randomized. In the matches between $A$ versus $B, C$ versus $D$, and $B$ versus $\mathrm{D}$, both the teams win equal number of matches and the net goal difference is zero in total. A always wins against $\mathrm{C}$ in the 

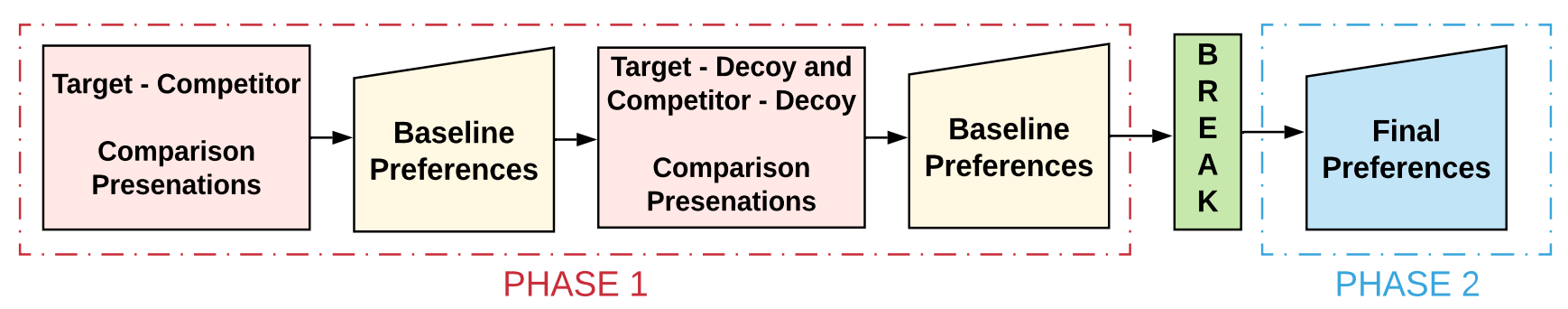

Figure 1: Longitudinal preferential choice experiment paradigm. In phase 1, subjects are shown all two-combination comparison presentations of target, competitor and decoy. Also, subjects are asked to enter their preferences frequently. These preference inputs constitute the baseline preferences or the initial preferences. Subjects are then asked for their final preferences in phase 2 with a compulsory break between phase 1 and phase 2.

matches between $\mathrm{A}$ and $\mathrm{C}$, and $\mathrm{A}$ scores significantly more goals than $\mathrm{C}$.

Experiment 1b Stimuli with two attributes were used. Each participant was shown animations of pair-wise horse races between four horses- A, B, C and D, along with food they consume after each race to get back the energy just spent. A frequency decoy was used. Block 1 consisted of sets of ten races of A versus $B$ and $C$ versus D. Block 2 had ten races of $A$ versus $C$. The order of the sets within each block and the matches within these sets were randomized. Each participant was assigned randomly to one of the following two types of choice contexts: two option choice contexts or three option choice contexts. In either context, all possible combination of choices were presented and the order of presentation of the choice sets were also randomized. In all the blocks, the participants entered their preference in the form of bets on each of the horses involved in the choice set. For each bet, the participant could use a maximum amount of $\$ 100$. In the pair-wise races of $\mathrm{A}$ and $\mathrm{B}, \mathrm{C}$ and $\mathrm{D}$, and $\mathrm{B}$ and $\mathrm{D}$, both the horses win equal number of races and the net wins or losses are the same. A wins against $\mathrm{C}$ eighty percent of the time in the matches between $\mathrm{A}$ and $\mathrm{C}$. In all of the races, the food stacks are either 4 or 6 , but in any match, they are the same for both the horses involved. The speed difference between the winning horse and loosing horse was kept constant throughout the experiment.

\section{Compromise Effect}

Experiment 2 Participants were shown pairwise comparisons of performance in bench-marking tests of three computer configurations - A, B and C. The configurations varied with respect to two attributes - Graphics processing(GPU) performance and General purpose computing(CPU) performance. All the three types of phase 1 comparisons consisted of a set of ten tests of general purpose computing tasks and a set of ten tests of graphics processing tasks. The order of presentation of the sets of each of these comparisons and the tests within each set were randomized. After every set, participants were asked for their preference in form of preference points (instead of \$) similar to experiment 1b. The partici- pants were first shown bench-marking tests between A and $\mathrm{B}$ to determine the target and the competitor. A beats $\mathrm{B}$ six times and $\mathrm{B}$ beats $\mathrm{A}$ four times in the general purpose computing tasks. A beats B four times and B beats A six times in the graphics processing tasks. If a participant preferred $\mathrm{A}$ more than $\mathrm{B}$, then $\mathrm{B}$ is the target, $\mathrm{A}$ is the competitor and $\mathrm{C}$ is the decoy. The dominant dimension would then be general computing task performance. Otherwise, $\mathrm{A}$ is the target, $\mathrm{B}$ is the competitor, $\mathrm{C}$ is the decoy and the dominant dimension is graphics processing task performance. Following this, the participants were shown target-decoy comparisons and competitor-decoy comparisons. In the target-decoy comparisons, the target wins seven times and the decoy wins three times in the dominant dimension. In the other dimension, the target wins four times and the decoy wins six times. In the competitor-decoy comparisons, the competitor wins eight times and the decoy wins two times in the dominant dimension. In the other dimension, the competitor wins 2 times and the decoy wins eight times. The order of presentation of the target-decoy comparisons and the competitor-decoy comparisons were randomized. In phase 2 , the participants were asked for their final preference for A, B and C with the choice set containing all the three options.

\section{Results}

McNemar's test was performed with the counts of the number of participants choosing the target over the competitor before the introduction of the decoy and after the introduction of the decoy respectively as the discordant cells (see table 1).

\section{Attraction Effect}

Experiment 1a Fifty five students (graduate and undergraduate) participated in the experiment for course credit. A strong attraction effect was observed (figure 3).

Experiment 1b One hundred students (graduate and undergraduate) participated in the experiment for course credit. Forty two students were randomly assigned to two option final choice contexts and fifty eight students were assigned three option final choice context.

Both the conditions failed to achieve statistical significance 


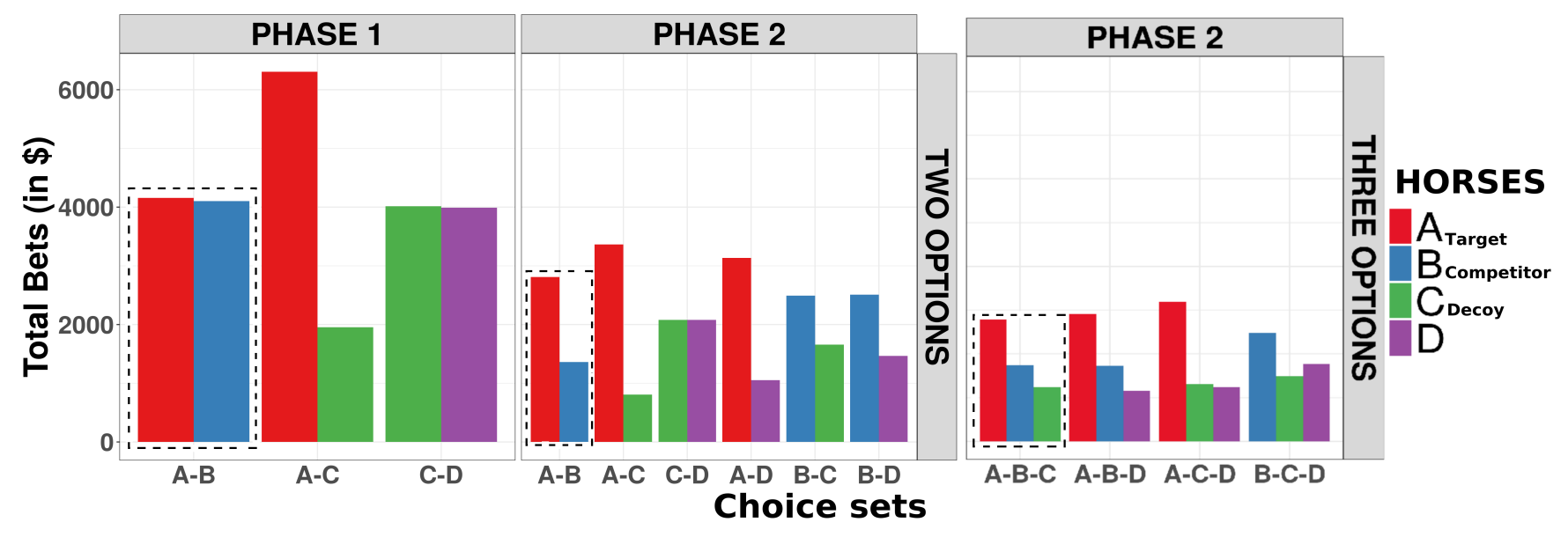

Figure 2: Results of experiment $1 \mathrm{~b}$. The first plot (from left) shows the participants' initial preference responses. The second and third plot shows their final preference responses when the choice context had two and three options each respectively. Note that initially, $A$ and $B$ are preferred almost equally. After the introduction of the decoy $C$ in phase $1, A$ is preferred over $B$ in the choice set containing $\{A, B\}$ or $\{A, B, C\}$ in phase 2 , suggesting a considerable attraction effect.

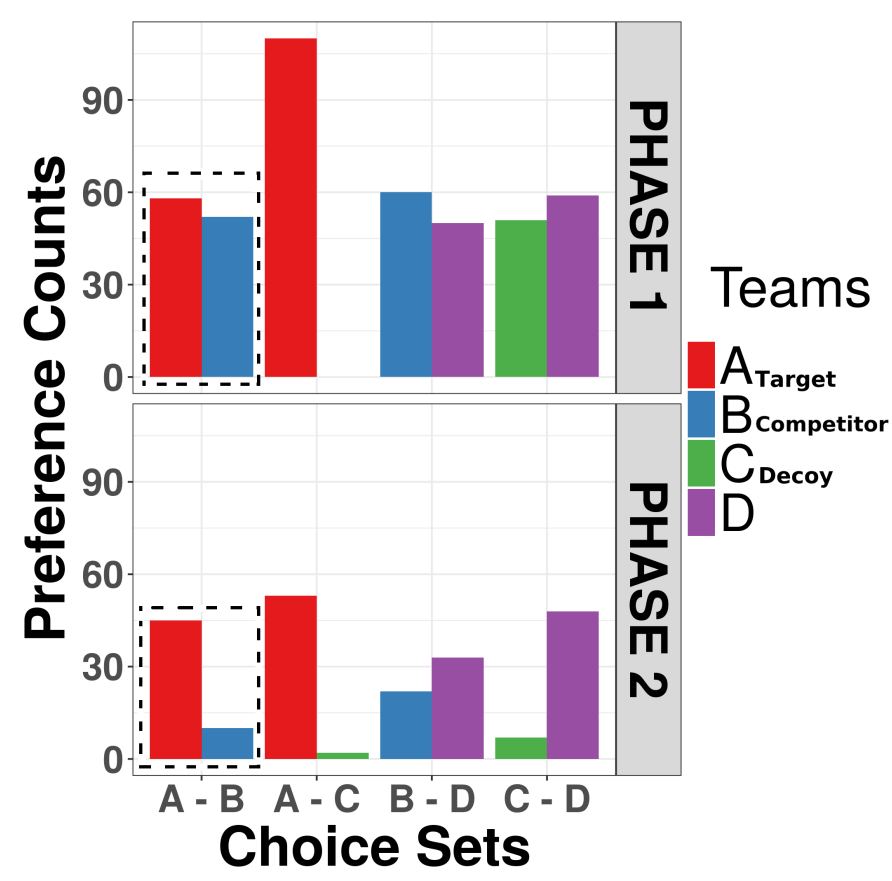

Figure 3: Results of experiment 1a. The first plot (from top) shows participants' initial preferences in block 1 and block 2. The second plot shows their final preferences. Note that initially, $A$ and $B$ are preferred almost equally. After the introduction of the decoy $C$ in phase $1, A$ is significantly preferred over $B$ in the choice set containing $\{A, B\}$ in phase 2 , indicating a strong attraction effect.

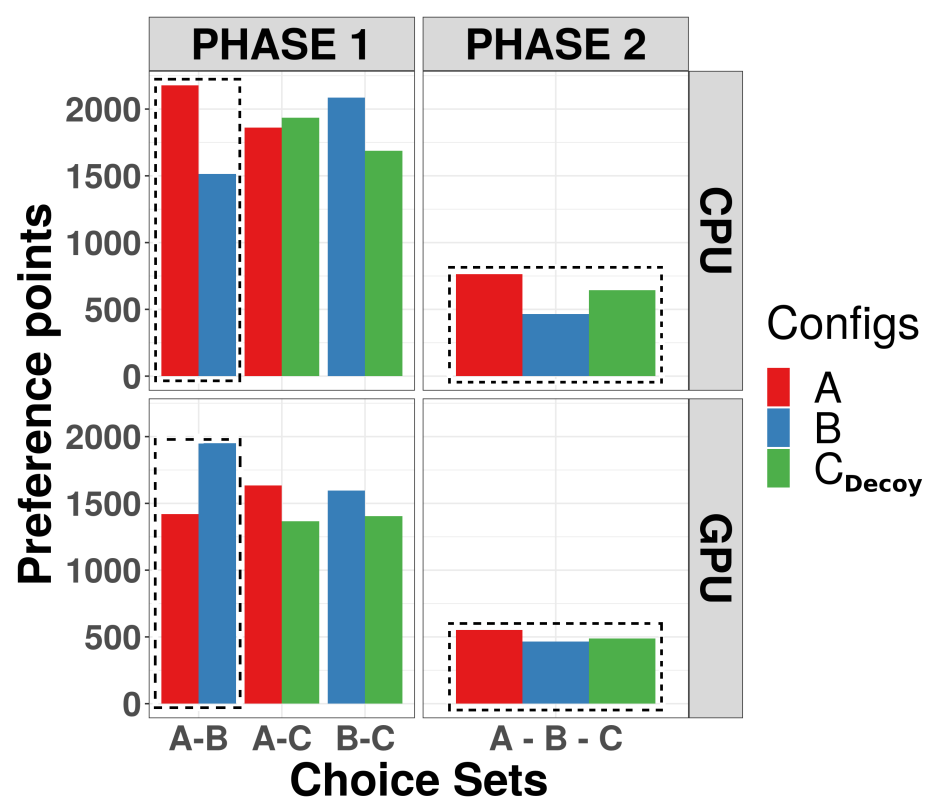

Figure 4: Results of experiment 2. The first row on the top corresponds to the case when CPU performance was the dominant dimension. The second row corresponds to the case when GPU performance was the dominant dimension. The first plot (from left) in each row shows the participants' initial preference responses. The second plot in each row shows their final preference responses when the choice context had three options. 
even though, the preference share for the target increased considerably compared to the competitor in phase 2 (figure 2).

\section{Compromise Effect}

Experiment 2 Thirty four students (graduate and undergraduate) participated in the experiment. A significant compromise effect was observed when the dominant dimension was graphics processing performance but the effect was not observed when the dominant dimension was general purpose computing performance (figure 4).

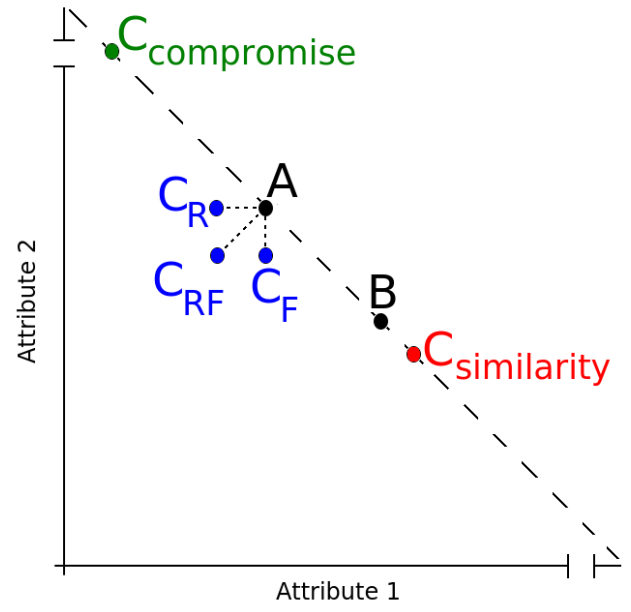

Figure 5: Different placements of decoy $C$, when $A$ and $B$ are the target and competitor options respectively. $A$ is better in one attribute dimension, $B$ is better in the other attribute dimension and there is no strong preference for either of them initially. In the case of the attraction effect, $C$ could be a range decoy $C_{R}$ or a frequency decoy $C_{F}$ or a range-frequency decoy $C_{R F}$. In the case of the similarity effect, the decoy $C_{\text {similarity }}$ is slightly different from and is equally attractive as the competitor option. In the compromise effect, the decoy $C_{\text {compromise }}$ is an extreme option and it makes the target option appear as a compromise between $C_{\text {compromise }}$ and $B$. In all cases, the introduction of the decoy increases the preference share of $A$ compared to $B$ (Trueblood et al., 2013).

\section{Discussion}

While a substantial attraction effect was seen, the results were inconclusive for the compromise effect. Nevertheless, the direction of preference change towards the target option suggests that prior history of choices is one variable that can influence preferences. We note that the original model we are testing (Srivastava \& Schrater, 2012, 2015) doesn't predict a compromise effect if the decoy is placed on the line (figure 5) and hence, it inherently requires such an asymmetry in the placement of decoy. The amount of decoy asymmetry and extremeness needed to elicit a strong compromise effect through this paradigm remains to be investigated. Also, the cover story of experiment 2 is from a very familiar domain

\begin{tabular}{||l||l|l|l||}
\hline \multirow{2}{*}{ Experiment } & \multicolumn{2}{|c|}{ Discordant Cells } & \multirow{2}{*}{-value } \\
\cline { 2 - 3 } & Before: $\mathrm{T}>\mathrm{C}$ & After: T $>\mathrm{C}$ & \\
\hline \hline 1a & 11 & 45 & $5.38 \times 10^{-6}$ \\
\hline 1b (Two) & 18 & 26 & 0.2912 \\
\hline 1b (Three) & 25 & 36 & 0.2004 \\
\hline 2 (CPU) & 0 & 4 & 0.125 \\
\hline 2 (GPU) & 0 & 12 & $4.883 \times 10^{-4}$ \\
\hline
\end{tabular}

Table 1: Columns 2 and 3 show the number of subjects who chose Target(T) over Competitor(C) before and after the introduction of the decoy respectively.

for the participants. It remains to be seen whether the differential elicitation of the compromise effect might be because of participants' priors on the domain.

Typically, in the preference reversal literature, multiattribute choices are presented to the participants with numerical values along the attribute dimensions and if the stimuli are perceptual, participants are asked for their preference on the same trial, thereby obviating any scope for inference from past choices. We demonstrated a novel experimental paradigm for preferential choice experiments. Participants were shown evidence of relative superiority of choices in different contexts along various attribute dimensions without resorting to presentation of numerical values along those dimensions. Based on the such a history, the participants supply their preferences for different choices frequently throughout the experiment. Our results suggest that preference inference is a realistic explanation for the occurrence of classic preference reversals.

\section{References}

Bhatia, S. (2013). Associations and the accumulation of preference. Psychological Review, 120(3), 522-543.

Hickson, L., \& Khemka, I. (2014). Chapter six - the psychology of decision making (Vol. 47). International Review of Research in Developmental Disabilities.

Kahneman, D., \& Tversky, A. (1979). Prospect theory: An analysis of decision under risk. Econometrica, 47(2).

Latty, T., \& Beekman, M. (2011). Irrational decisionmaking in an amoeboid organism: transitivity and contextdependent preferences. Proceedings of the Royal Society.

Rigoli, F., Mathys, C., Friston, K. J., \& Dolan, R. J. (2017). A unifying bayesian account of contextual effects in valuebased choice. PLOS: Computational Biology, 13(10).

Slovic, P. (1995). The construction of preference. American psychologist, 50(5), 364-371.

Srivastava, N., \& Schrater, P. (2015). Learning what to want: Context-sensitive preference learning. PLOS ONE, 10(10).

Srivastava, N., \& Schrater, P. R. (2012). Rational inference of relative preferences. NIPS.

Trueblood, J. S., Brown, S. D., Heathcote, A., \& Busemeyer, J. R. (2013). Not just for consumers: Context effects are fundamental to decision making. Association for Psychological Science, 24(6), 901-908. 